\title{
The Relationship between Overactive Bladder and Sexual Activity in Women
}

\author{
Ankur S. Patel, Margie L. O'Leary, Robert J. Stein, Wendy W. Leng, Michael B. Chancellor, \\ Subodh G. Patel, Diane Borello-France
}

Department of Urology (ASP, MLO, RJS, WWL, MBC), University of Pittsburgh Medical Center, Pittsburgh, Pennsylvania, Uniontown Hospital (SGP), Uniontown, Pennsylvania, and Department of Physical Therapy (DBF), Duquesne University, Pittsburgh, Pennsylvania, USA

\begin{abstract}
Purpose: We assessed the relationships between bladder symptoms, demographic, and medical history variables and sexual dysfunction in women with overactive bladder (OAB) disorder.

Materials and Methods: Seventy-eight women diagnosed with OAB completed self-administered questionnaires related to overall heath status, bladder function, and sexual function. Data were compiled for questionnaire responses, and multivariate logistic regression analyses were performed to determine predictors of sexual dysfunction.

Results: Bothersome bladder symptoms were reported by $\geq 60 \%$ of the sample. Sixty-percent of the sample was sexually active in the past month. Difficulty with sexual arousal, orgasm, and sexual enjoyment were reported by about $25 \%$ of the women. Sexual partner status was the best predictor of sexual arousal, orgasm, and sexual enjoyment. Menopausal status emerged as an important predictor of arousal and sexual enjoyment.

Conclusion: The majority of women with symptoms of OAB viewed these symptoms as bothersome. However, the extent of symptom bother did not predict aspects of female sexual dysfunction (FSD). Instead, menopausal and partner status emerged as the best predictors of FSD in our sample.
\end{abstract}

Key words: urination disorders; female; overactive bladder; sexual dysfunction; menopause Int Braz J Urol. 2006; 32: 77-87

\section{INTRODUCTION}

Although reported estimates of sexual dysfunction are higher for women than men (43\% versus $31 \%$, respectively), fewer treatment options for female sexual dysfunction (FSD) exist (1). As treatments for male sexual dysfunction have become safer and more effective, both individuals and the medical community are seeking beneficial therapies for FSD.

According to the World Health Organization (2) and the Diagnostic and Statistical Manual of Mental Disorders (3), FSD is classified into disorders of desire (hypoactive sexual desire, sexual aversion), arousal, orgasm, and pain (dyspareunia and vaginismus). Physiologic, iatrogenic, and psychological factors place women at risk for developing FSD. Physiologic and iatrogenic factors include abdominal surgery (e.g., hysterectomy), menopause, smoking, spinal cord injury, and certain medications (e.g., birth control pill, antipsychotics, antihypertensives, antidepressants, etc.). Psychologic factors include anxiety, depression, negative body perception, history of physical or emotional abuse, and stress.

Overactive bladder (OAB) disorder may also contribute to the development of FSD (4). According to the International Continence Society (5), OAB dis- 
order is classified as a symptom syndrome suggestive of lower urinary tract dysfunction. Symptoms of $\mathrm{OAB}$ include urinary urgency, urinary frequency, and urge urinary incontinence. Overactive bladder drastically reduces quality of life by its negative influence on a person's ability to work, travel, sleep, exercise, socialize, and participate in intimate relationships (4).

In current health care practices, family physicians, not specialists, are most likely to manage the treatment of OAB in women. Discussions regarding bladder symptoms between patients and their physicians are occurring more frequently. In addition, family care physicians have enhanced access to diagnostic testing and safer and more effective medications to treat $\mathrm{OAB}$.

Conversely, discussion and assessment of female sexual health occurs much less frequently. This may be partially attributed to the opinion that the origin of FSD is more psychological than physical. Women might also believe that sexual health is a topic that should discussed with a specialist, such as her gynecologist. Likewise, if her family physician fails to ask about sexual health, she may avoid discussion based on the belief that her family physician is uncomfortable with the topic. Limited familiarity with sexual health assessments and treatment options may further prevent the physician from discussing FSD with a patient (6). Discussion regarding sexually related issues are highly challenging within primary care because of their sensitivity, complexity, and constraints of time and expertise (7).

On the contrary, men have many options when seeking treatment for erectile dysfunction. They may actually arrive at their primary-care appointment seeking treatments as a result of increased direct-to-consumer advertising by pharmaceutical companies. In the near future, women may be more willing to discuss their sexual health as FDA approved medications to treat FSD enter the market.

In the case of FSD, family physicians lack easy access to physiological measures of patients' experiences. Symptom-related and health-related quality of life (HRQOL) measurements may provide useful information to guide treatment selection and to evaluate treatment effectiveness.
Since female sexual health problems are often undiagnosed and untreated, our first study aim was to enhance awareness by describing sexual health in a sample of women with OAB using symptom-related and HRQOL measures. Because control of bladder and sexual function occurs through shared neurologic pathways, we also aimed to investigate the extent that bladder dysfunction and demographic and medical history variables predicted sexual dysfunction. We hypothesized that the likelihood of sexual dysfunction would be associated with higher levels of distress related to bladder symptoms.

\section{MATERIALS AND METHODS}

One hundred fifty female patients from a suburban general urology practice, between the ages of 35-70 years, with clinically documented OAB symptoms (within a 12-month period) were asked to participate in this study. Data were obtained via self-administered questionnaires that were mailed to participants. The questionnaires included: the Urinary Distress Inventory-Short Form (UDI), the Personal Experiences Questionnaire (PEQ), and a demographic and medical history questionnaire. The respondents completed all questionnaires anonymously.

The UDI assesses the presence and degree of bother associated with bladder symptoms, including urge incontinence, stress incontinence, small volume leakage, difficulty emptying the bladder, and pain in the lower abdominal area (8). Psychometric properties including internal consistency, concurrent (with UDI, long version) and construct validity, and sensitivity to change of the UDI have been reported (8).

The PEQ includes a subset of eleven items derived from the McCoy Female Sexuality Questionnaire (9). Items included sexual frequency, sexual arousal, frequency of orgasm, pain during intercourse, feelings towards partner, and partner problems. Despite limited information on its reliability and validity characteristics, we chose the PEQ for its brevity, ease of completion, and face validity.

Women also completed a demographic and medical history questionnaire. This questionnaire elicited descriptive information regarding age, marital 
status, race, disability, menopausal status, sexual partner, depression, and past surgeries.

\section{Data Analysis}

Means, standard deviations, and percentages were calculated in order to describe the sample with respect to demographic and medical history variables. Percentages were calculated to describe responses to the UDI and PEQ questionnaires.

For statistical analyses, the questionnaire responses were collapsed into smaller categories. Urogenital Distress Inventory responses "not at all", "slightly", "moderately", and "greatly" were collapsed into "not at all bothered" and "bothered" categories. The PEQ includes a semantic differential rating scale, with assigned values from 1 ("not at all") to 6 ("a great deal"). For our analyses, values 2 and 3 were collapsed to create a "sometimes" category, and values 4,5 , and 6 were collapsed to create "a great deal" category.

Multivariate logistic regression (MLR) was used to analyze factors associated with the likelihood of sexual dysfunction. Potential factors (age, menopausal status, depression, available sexual partner, bladder symptom-related distress) identified using univariate logistic regression analyses as having a relationship $(\mathrm{p} \leq .10)$ with sexual function were used in the MLR procedure to identify the best subset of variables associated with sexual dysfunction.

\section{RESULTS}

\section{Patient Description}

One hundred women returned the completed questionnaires. Twenty-two indicated a medical history of neurologic disease or injury. Data from these 22 women were excluded, leaving usable questionnaire data from 78 women. The mean sample age was $50 \pm 6$ years, and $87 \%$ of the women were Caucasian. Fifty percent of the respondents were married, $14.1 \%$ were single, $12.8 \%$ were divorced, and $11.5 \%$ were widowed. Ninety-six percent of the sample was heterosexual and $72 \%$ indicated that they had a current sexual partner.

The percent of women indicating co-morbid medical conditions is shown in Figure-1. Hypertension and depression were the most common co-mor-

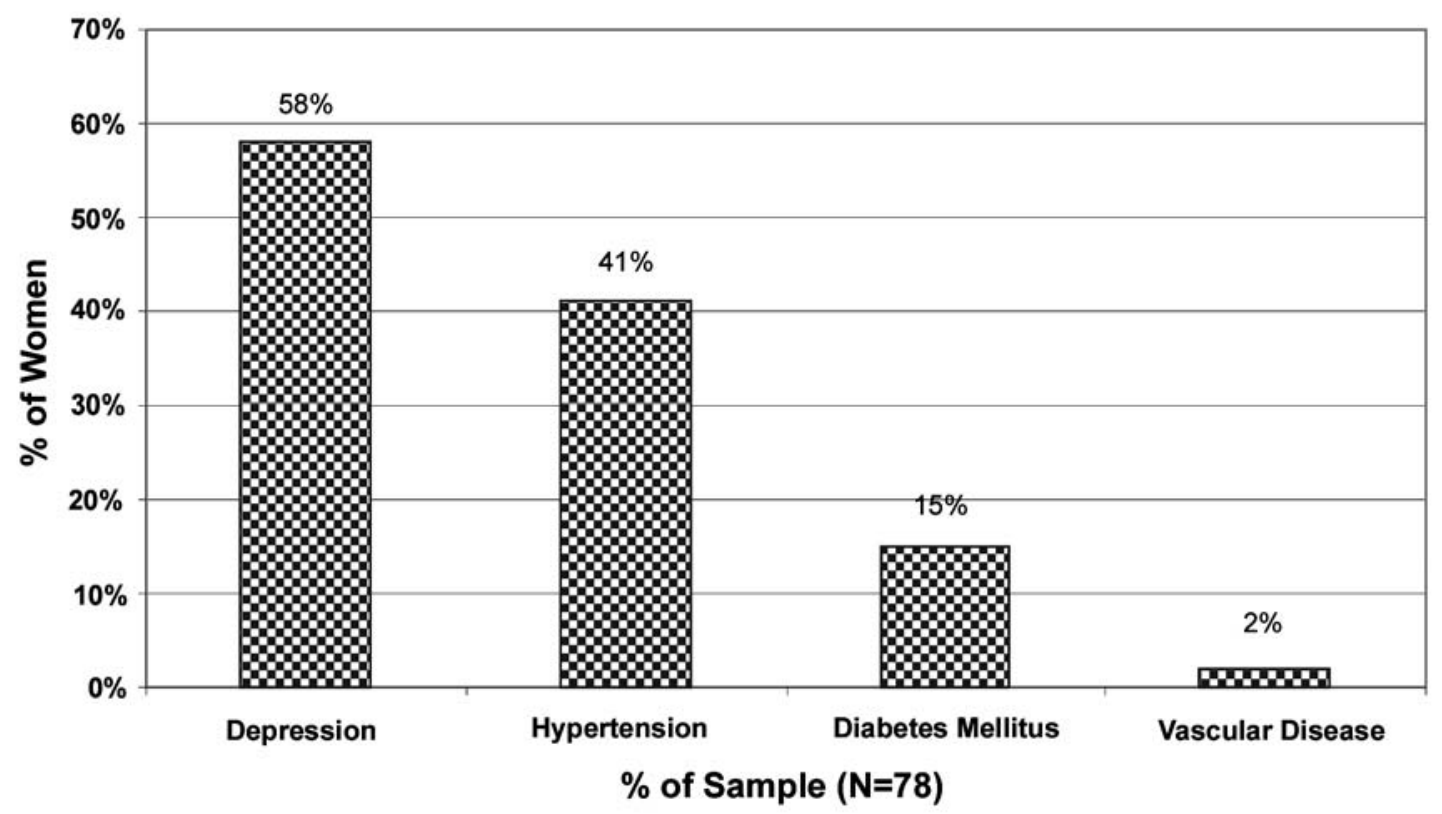

Figure 1 - Percentage of women reporting co-morbid medical conditions. 
bid conditions. Forty-seven percent of the sample indicated current treatment for depression.

\section{Bladder Symptom and Sexual Function Question Results}

The distribution of reported bladder symptoms are shown in Figure-2. Frequent urination, urge incontinence, small volume leaks, and stress incontinence were all reported by $\geq 60 \%$ of the sample. Difficulty voiding and pain in the lower abdominal/ genital area were reported by less than $40 \%$ of the sample. Across all symptoms, $99-100 \%$ of the women reporting a symptom indicated that the symptom was bothersome. Responses to the sexual function questionnaire items are shown in Figure-3. Inabilities to become sexually aroused, experience orgasm, and enjoy sex were reported by $24 \%, 27 \%$, and $27 \%$ of the women, respectively. Twenty-five percent of the women were dissatisfied with their partner as a lover. Thirty-six percent of respondents had a partner who experienced difficulty in sexual performance.
Figures-4 and 5, show the frequency of sexual thoughts/fantasies and sexual activity responses. In the past month, 29\% had not experienced sexual thoughts or fantasies and $40 \%$ of the respondents had not engaged in sexual activity.

\section{Univariate Logistic Regression Analyses}

Tables- 1 to 3 show the results of the univariate logistic regression analyses. Sexual partner status was the only independent variable that displayed a statistically significant association with arousal $(\mathrm{p}<0.05)$. The association with menopausal status approached statistical significance $(\mathrm{p}=0.08)$. These variables were retained for a MLR model to identify the best subset of variables indicative of a dysfunction in sexual arousal.

Sexual partner status was the only independent variable that displayed a statistically significant $(p<0.05)$ relationship with orgasm. Women lacking a sexual partner were almost 22 times more likely to experience anorgasm compared to women with a sexual partner (see Table-4).

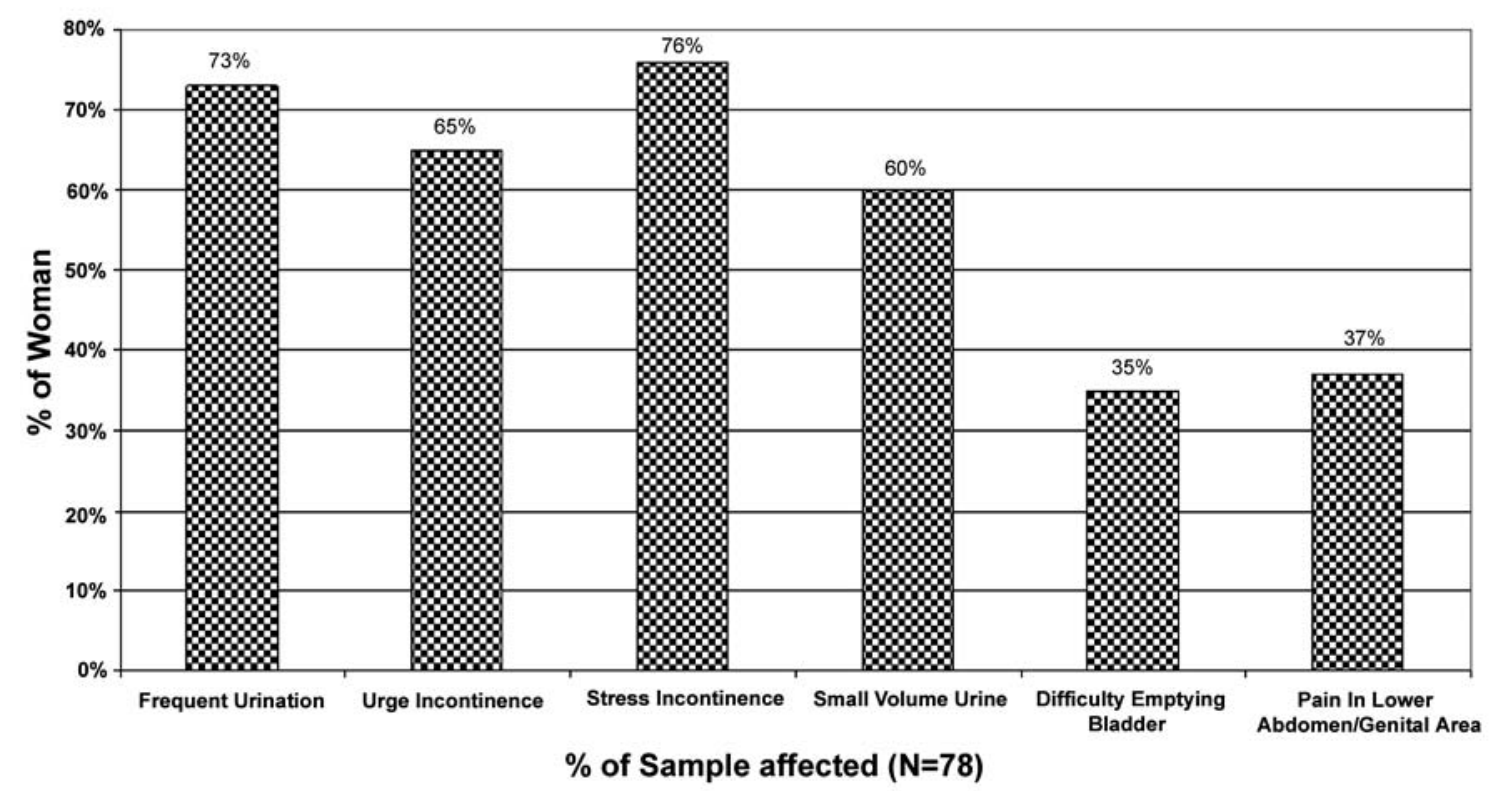

Figure 2 - Percentage of women indicating specific bladder symptoms. 


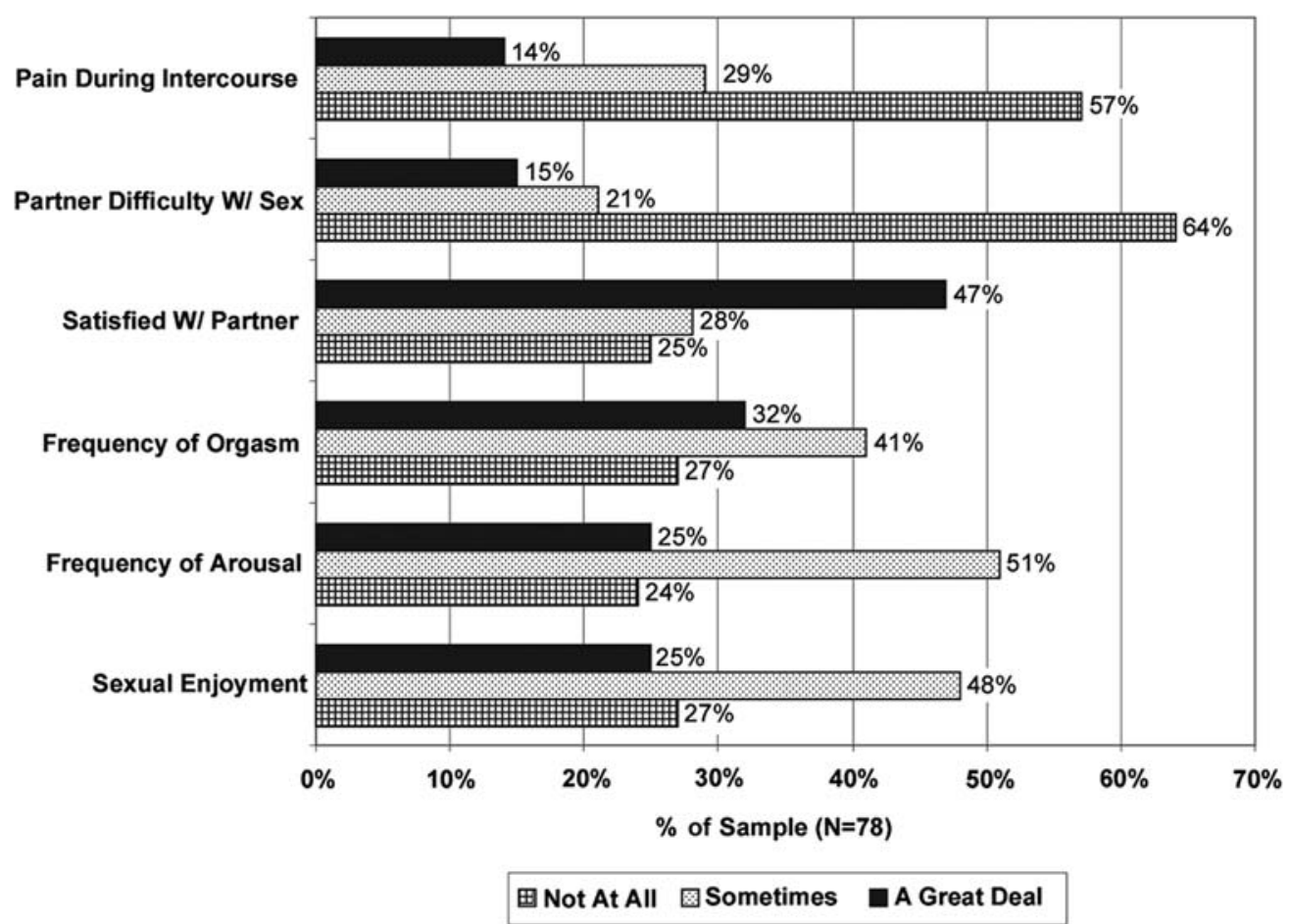

Figure 3 - Responses to the Personal Experiences Questionnaire. The bars indicate the percentage of women answering either "not at all", "sometimes", or "a great deal" to sexual function questions.

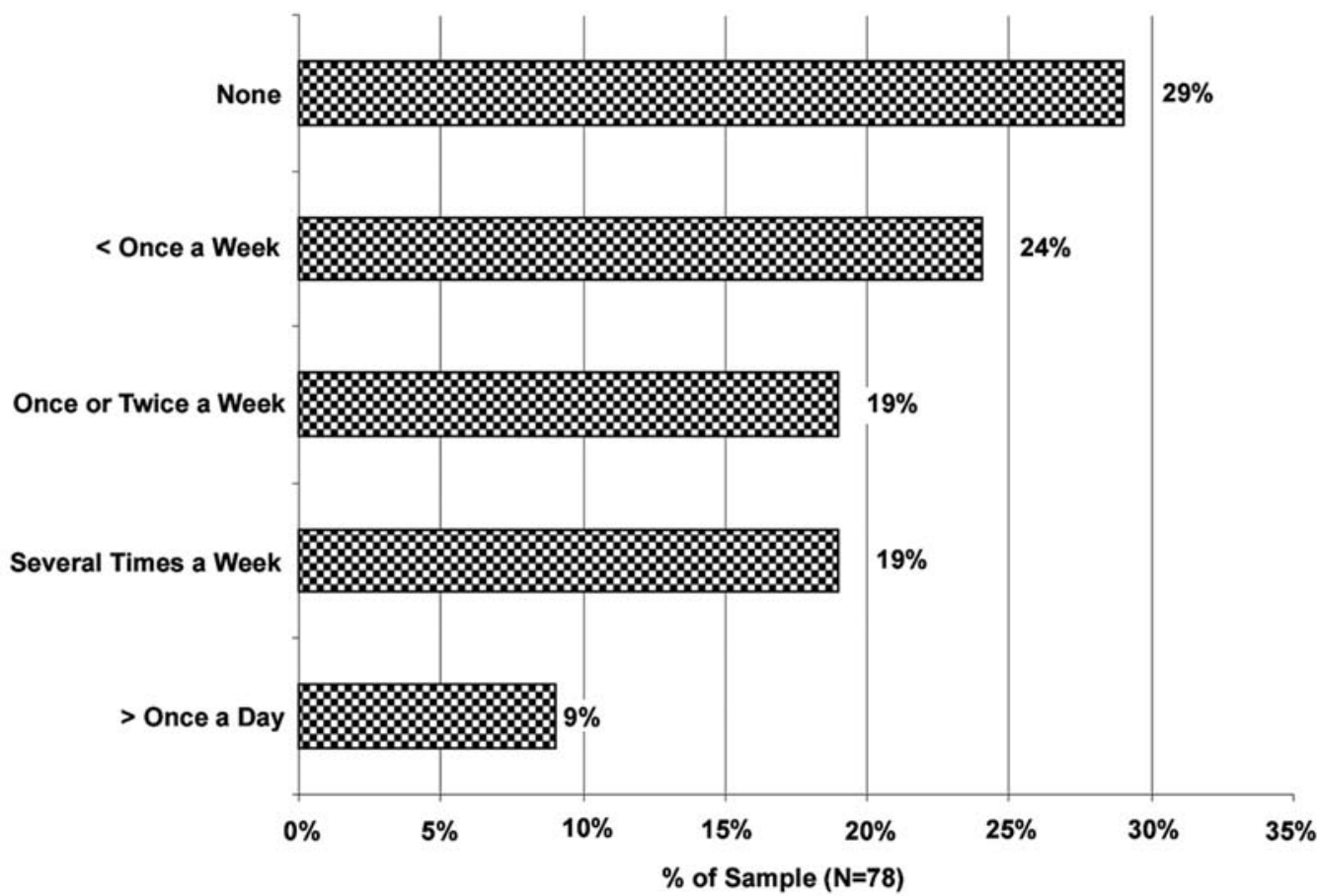

Figure 4-Responses to the Personal Experiences Questionnaire question: In the past month, how often have you experienced sexual thoughts or fantasies? 


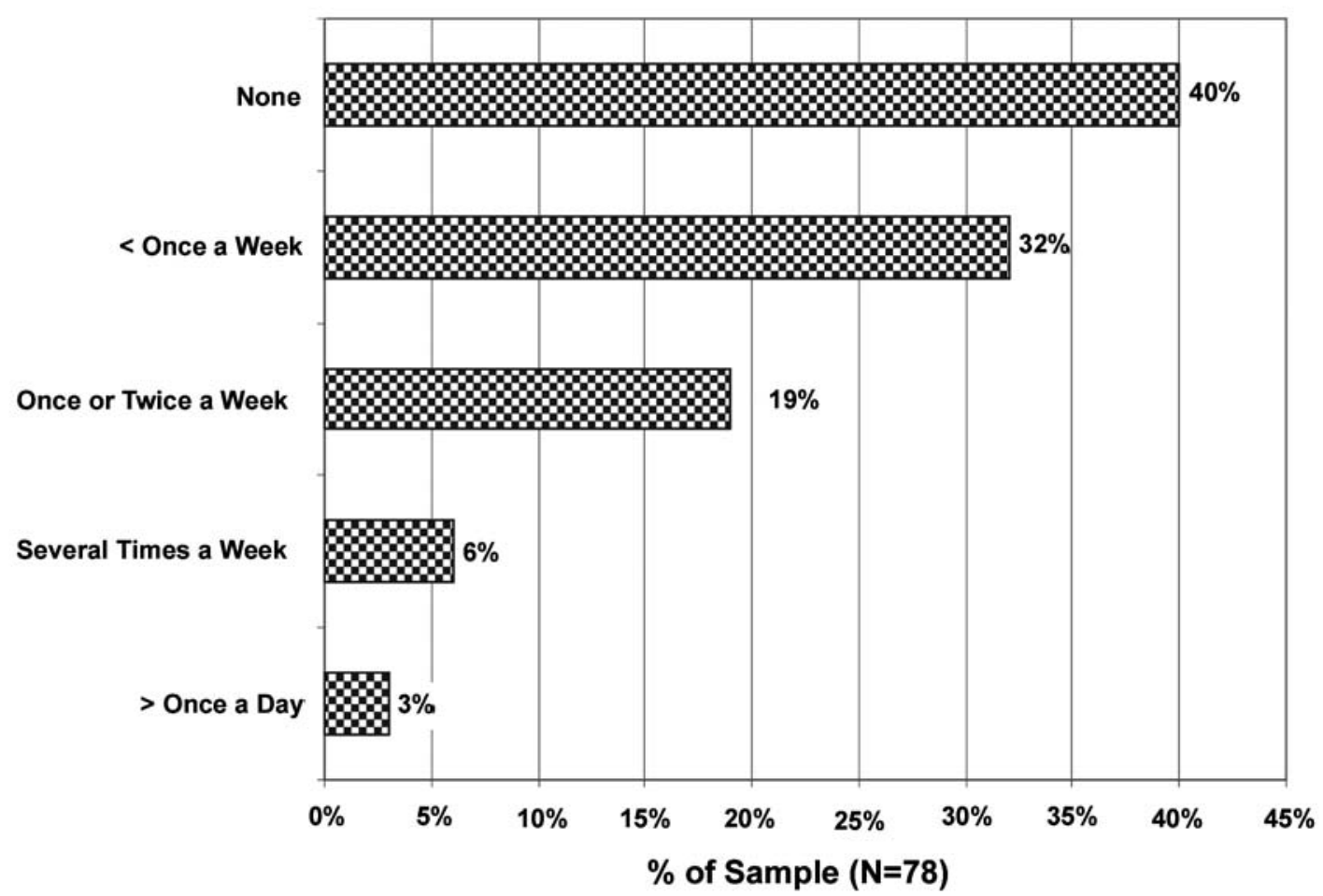

Figure 5 - Responses to the Personal Experiences Questionnaire question: In the past month, how often have you experienced sexual activity?

Independent variables showing associations with sexual enjoyment included sexual partner status $(p=0.00)$, menopausal status $(p=0.09)$, and urge incontinence $(p=0.10)$. These 3 variables were retained for the MLR model to identify the best subset of predictors of low sexual enjoyment.

\section{Multivariate Logistic Regression Analyses}

Tables- 5 and 6 show the odds ratios and confidence intervals for each independent variable tested in the models predicting sexual arousal and enjoyment dysfunction. The model for predicting sexual arousal dysfunction (Table-5) showed statistical sig-

Table 1 - Results of univariate logistic analysis with dependent variable: Arousal.

\begin{tabular}{lccc}
\hline Independent Variable & $\mathbf{- 2 ~ L o g ~ L i k e l i h o o d ~}$ & Chi-Square & Significance \\
\hline Leak urge & 17.42 & 2.54 & 0.28 \\
Distress related to urge incontinence & 16.78 & 3.61 & 0.16 \\
Frequent urination & 17.28 & 2.83 & 0.24 \\
Distress related to frequent urination & 14.91 & .95 & 0.62 \\
Leak with physical activity & 14.83 & .247 & 0.88 \\
Distress related to stress incontinence & 10.17 & 1.80 & 0.41 \\
Menopause & 19.68 & 5.10 & 0.08 \\
Sexual partner & 33.78 & 20.09 & 0.000 \\
\hline
\end{tabular}

Note: all independent variables were dichotomous: sexual partner and menopause (no/yes), presence of bladder symptom (yes/no), distress related to bladder symptoms (not bothered/bothered). 
Table 2 - Results of univariate logistic analysis with dependent variable: Orgasm.

\begin{tabular}{lccc}
\hline Independent Variable & $\mathbf{- 2}$ Log Likelihood & Chi-Square & Significance \\
\hline Leak urge & 17.37 & 2.44 & 0.30 \\
Distress related to urge incontinence & 14.34 & .90 & 0.64 \\
Frequent urination & 17.27 & 2.74 & 0.26 \\
Distress related to frequent urination & 16.81 & 2.94 & 0.23 \\
Leak with physical activity & 15.13 & .49 & 0.78 \\
Distress related to stress incontinence & 10.28 & 1.90 & 0.39 \\
Menopause & 17.78 & 3.04 & 0.22 \\
Sexual partner & 32.30 & 18.58 & 0.000 \\
\hline
\end{tabular}

Note: all independent variables were dichotomous: sexual partner and menopause (no/yes), presence of bladder symptom (yes/no), distress related to bladder symptoms (not bothered/bothered).

Table 3 - Results of univariate logistic analysis with dependent variable: Enjoyment.

\begin{tabular}{|c|c|c|c|}
\hline Independent Variable & -2 Log Likelihood & Chi-Square & Significance \\
\hline Leak urge & 19.471 & 4.62 & 0.10 \\
\hline Distress related to urge incontinence & 16.66 & 3.45 & 0.18 \\
\hline Frequent urination & 18.10 & 3.53 & 0.17 \\
\hline Distress related to frequent urination & 16.05 & 2.13 & 0.34 \\
\hline Leak with physical activity & 16.21 & 1.67 & 0.43 \\
\hline Distress related to stress incontinence & 10.50 & 2.10 & 0.35 \\
\hline Menopause & 19.50 & 4.82 & 0.09 \\
\hline Sexual partner & 30.40 & 16.49 & 0.000 \\
\hline
\end{tabular}

Note: all independent variables were dichotomous: sexual partner and menopause (no/yes), presence of bladder symptom (yes/no), distress related to bladder symptoms (not bothered/bothered).

nificance. Sexual partner status was most influential in predicting the inability to become sexually aroused. Women without a sexual partner were almost 22 times more likely to report an inability to become sexually aroused compared to women with a sexual partner. Neither sexual partner nor menopausal status emerged as predictors of arousal "sometimes".

The model for predicting the failure to enjoy sexual activities also showed statistical significance (Table-6). Lacking sexual partner was the best predictor of the failure to enjoy sexual activities. Women without a partner were over 14 times more likely to report that sexual activities were "not at all enjoyable" compared to women with a sexual partner. In addition, postmenopausal women were al- most 5 times more likely to report sexual activities that were "not enjoyable at all" compared to premenopausal women.

\section{COMMENTS}

\section{Prevalence of Bladder Symptoms and Sexual Dysfunction}

The primary aim of this study was to describe sexual health in a sample of women with OAB. Female sexual dysfunction and $\mathrm{OAB}$ syndrome share many risk factors including age, medications, smoking, hormonal changes, hysterectomy, and uterine prolapse (10). In this study, women who reported 
Table 4-Odds of anorgasm by sexual partner status.

\begin{tabular}{lcccc}
\hline Orgasm & Independent Variable & Significance & Odds Ratio & 95\% CI \\
\hline Not at all & No sexual partner & 0.00 & 21.73 & $4-125$ \\
Sometimes & No sexual partner & 0.13 & 3.81 & $0.67-21.74$ \\
\hline
\end{tabular}

Reference group, women who experience orgasm "very often". Note: model significant at the level of 0.00; -2 log likelihood = 46.27; $\chi^{2}=25.16$.

Table 5 - Multivariate logistic regression results for factors associated with arousal.

\begin{tabular}{llccl}
\hline Arousal & Independent Variable & Significance & Odds Ratio & 95\% CI \\
\hline \multirow{2}{*}{ Not at all } & Menopause & 0.070 & 4.33 & $0.88-21.15$ \\
\multirow{2}{*}{ Sometimes } & No sexual partner & 0.000 & 21.74 & $4.22-111$ \\
& Menopause & 0.059 & 3.08 & $0.95-9.9$ \\
& No sexual partner & 0.408 & 2.0 & $0.39-10.41$ \\
\hline
\end{tabular}

Reference group, women who experience arousal "very often". Note: model significant at the level of 0.00; -2 log likelihood = 46.27; $\chi^{2}=25.16$.

Table 6 - Multivariate logistic regression results for factors associated with enjoying sex.

\begin{tabular}{llccl}
\hline Enjoy Sex & Independent Variable & Significance & Odds Ratio & 95\% CI \\
\hline \multirow{2}{*}{ Not at all } & Urge incontinence & 0.925 & 1.07 & $0.27-4.22$ \\
& Menopause & 0.049 & 4.62 & $1.004-21.19$ \\
& No sexual partner & 0.001 & 14.93 & $3.06-71.4$ \\
Sometimes & Urge incontinence & 0.094 & 2.89 & $0.833-10.10$ \\
& Menopause & 0.199 & 2.17 & $0.67-7.05$ \\
& No sexual partner & 0.493 & 1.78 & $0.34-9.35$ \\
\hline
\end{tabular}

Reference group, women who enjoy sex “very often”. Note: model significant at the level of $0.00 ;-2$ log likelihood $=61.85 ; \chi^{2}=24.80$.

symptoms of urinary frequency, stress, and urge incontinence indicated that these symptoms were indeed bothersome. Others have reported the impact of $\mathrm{OAB}$ on quality of life. Women with urinary incontinence feel social isolation, depressive symptoms, anxiety, humiliation, and loss of self-confidence (11). Approximately $25 \%$ of our sample of women with $\mathrm{OAB}$ identified some form of sexual dysfunc- tion. In addition, $40 \%$ indicated that they did not engage in sexual activity during the month surveyed. Although women in our study reported both bladder and sexual dysfunction, we did not find statistically significant associations between the two disorders. However, Salonia (12) demonstrated that sexual function is negatively affected in women with bladder dysfunction. 


\section{Pain and Sexual Function}

Almost $40 \%$ of our sample indicated that they experienced bothersome pain in their abdominal/genital area. In our study, we did not find abdominal/genital pain a significant predictor of sexual dysfunction. Based on findings of a national study including 1749 women, Laumann et al. reported that urinary tract symptoms contributed to arousal and pain disorders in women (1). Meana et al., reported that women with dyspareunia had greater sexual dysfunction and psychologic distress compared to age-matched women without dyspareunia (13). The disparity between these published studies and our results could reflect a difference in the primary symptom complaint of our sample. A urologist was examining the women surveyed for the primary complaint of $\mathrm{OAB}$, not abdomi$\mathrm{nal} / \mathrm{pelvic}$ pain.

\section{Menopausal Factors and Sexual Function}

Gynecologic changes occurring during menopause include vaginal atrophy and dryness, urinary incontinence, and infections. A loss of pelvic floor muscle tone or diminished capability of the intrinsic urethral sphincter, which can cause incontinence and decreased maximum urethral closing pressures may also occur (14). Decreases in sexual activity are also associated with estrogen level decline and testosterone level fluctuation (15).

Our results indicated that menopausal status was a statistically significant predictor of sexual enjoyment. Postmenopausal women in our study were almost 5 times more likely to report "not enjoyable" sexual activities compared to pre-menopausal women. Dennerstein et al. also demonstrated significant changes in sexual function during menopause related to sexual desire, frequency, response, and feelings toward the sexual partner (16). Likewise, Blumel et al. showed that Chilean women, aged 40-64, reported decreased or absent sexual activity as they transitioned to menopause (17). Even when age was held constant, menopausal symptoms were determined to be significant factors in ceasing sexual activity.

Long-term absence of sexual function accompanied by a lack of estrogen post-menopause can cause vaginal atrophy and dryness. These physiologi- cal changes make sexual activity difficult as vaginal stretching capacity is diminished.

Post-menopausal sexual function may also be limited by depressive symptoms. Freeman observed that increases in depressive symptoms occurred as women transitioned into menopause and demonstrated fluctuating estradiol levels (18). Although we did not find depression to be a predictor of sexual dysfunction, $47 \%$ of our sample did indicate that they were receiving treatment for depression.

\section{Partner Effect}

Our data showed that sexual partner status was the best predictor of sexual arousal, orgasm, and sexual enjoyment. Twenty-eight percent of our sample did not have a current sexual partner. Reasons for lacking a partner can include death of spouse, divorce, history of depression, previous negative sexual experience, career aspirations, or competing family demands. One limitation of our study is that we did not elicit specific reasons for lacking a sexual partner from our sample. Such reasons could have confounded our results by inflating the importance of sexual partner status as a predictor of sexual function. For example, social and family structure changes that occur in the middle-age years can create changes in daily life activities. The additional responsibility of caring for elderly parents or grandchildren could impact sexual function by limiting time to devote toward partner relationships.

Another limitation of our study is that we did not thoroughly explore the impact of other partner variables on FSD. Thirty-five percent of our sample indicated that they had a partner who experienced difficulty in sexual performance and $24 \%$ admitted that they were dissatisfied with their partner as a lover. We did not elicit from these women the reasons for their dissatisfaction. Aside from partner difficulties, the quality of the sexual experience may have been an important factor to consider. Young showed that the consistent ability of both partners to achieve orgasm had an impact on female sexual satisfaction (19). Pre-coital caressing and frequent coitus have also been reported by women to be important to sexually satisfying marital relationships (20). Because partner status emerged as such a strong predictor of sexual func- 
tion, future research should include a thorough exploration of partner-related variables.

\section{CONCLUSION}

Although this study sample consisted of women seeking care from a urologist, our results are relevant to the family practice field. Family physicians are assessing and treating bladder symptoms at increasingly high rates. In addition, FSD is still not a topic commonly discussed in a primary care visit. Since FSD directly impacts quality of life, family care physicians should discuss FSD with all female patients. Based on our results, particular attention should be directed to menopausal women and women lacking a sexual partner as they are more likely to be experiencing sexual dysfunction.

\section{ACKNOWLEDGEMENT}

This work was supported by the Center of Urology Research Excellence-Spinal Cord Injury (CURE-SCI)

\section{CONFLICT OF INTEREST}

None declared.

\section{REFERENCES}

1. Laumann EO, Paik A, Rosen RC: Sexual dysfunction in the United States: prevalence and predictors. JAMA. 1999; 281: 537-44. Erratum in: JAMA 1999; 281: 1174.

2. World Health Organization: ICD-10: International Statistical Classification of Diseases and Related Health Problems, ed. 10. Geneva, World Health Organization. 1992.

3. American Psychiatric Association. DSM-IV: Diagnostic and Statistical Manual of Mental Disorders. Washington, American Psychiatric Association. 1994.

4. Jackson $\mathrm{S}$ : The patient with an overactive bladdersymptoms and quality-of-life issues. Urology. 1997; 50 (6A Suppl): 18-22; discussion 23-4.
5. International Continence Society: First report on the standardization of terminology of lower urinary tract function. Br J Urol. 1976; 48: 39-42.

6. Sarkadi A, Rosenqvist U: Contradictions in the medical encounter: female sexual dysfunction in primary care contacts. Fam Pract. 2001; 18: 161-6.

7. Gott M, Galena E, Hinchliff S, Elford H: "Opening a can of worms": GP and practice nurse barriers to talking about sexual health in primary care. Fam Pract. 2004; 21 : 528-36.

8. Uebersax JS, Wyman JF, Shumaker SA, McClish DK, Fantl JA: Short forms to assess life quality and symptom distress for urinary incontinence in women: the Incontinence Impact Questionnaire and the Urogenital Distress Inventory. Continence Program for Women Research Group. Neurourol Urodyn. 1995; 14: 131-9.

9. McCoy NL: The McCoy Female Sexuality Questionnaire. Qual Life Res. 2000; 9: 739-45.

10. Bachmann GA, Leiblum SR: The impact of hormones on menopausal sexuality: a literature review. Menopause. 2004 ; 11: 120-30.

11. Temml C, Haidinger G, Schmidbauer J, Schatzl G, Madersbacher S: Urinary incontinence in both sexes: prevalence rates and impact on quality of life and sexual life. Neurourol Urodyn. 2000; 19: 259-71.

12. Salonia A, Munarriz RM, Naspro R, Nappi RE, Briganti A, Chionna R, et al.: Women's sexual dysfunction: a pathophysiological review. BJU Int. 2004; 93: 1156-64.

13. Meana M, Binik YM, Khalife S, Cohen DR: Biopsychosocial profile of women with dyspareunia. Obstet Gynecol. 1997; 90: 583-9.

14. Goldberg RP, Sand PK: Pathophysiology of the overactive bladder. Clin Obstet Gynecol. 2002; 45: 18292.

15. Gracia CR, Sammel MD, Freeman EW, Liu L, Hollander L, Nelson DB: Predictors of decreased libido in women during the late reproductive years. Menopause. 2004 ; 11: 144-50.

16. Dennerstein L, Dudley E, Burger H: Are changes in sexual functioning during midlife due to aging or menopause? Fertil Steril. 2001; 76: 456-60.

17. Blumel JE, Castelo-Branco C, Cancelo MJ, Romero $\mathrm{H}$, Aprikian D, Sarra S: Impairment of sexual activity in middle-aged women in Chile. Menopause. 2004; 11: 78-81.

18. Freeman EW, Sammel MD, Liu L, Gracia CR, Nelson DB, Hollander L: Hormones and menopausal status as predictors of depression in women in transition to menopause. Arch Gen Psychiatry. 2004; 61: 62-70. 
19. Young M, Denny G, Young T, Luquis R: Sexual satisfaction among married women age 50 and older. Psychol Rep. 2000; 86: 1107-22.
20. Zhou M: A survey of sexual states of married, healthy, reproductive age women. J Psychol Human Sex. 1993; 6: 15-28.

Correspondence address:

Dr. Michael B. Chancellor

Kaufmann Building

3471 Fifth Avenue Suite 700

Pittsburgh, Philadelphia, 15213, USA

Fax: + 1412 692-4101

E-mail: chancellormb@upmc.edu 